\title{
Three-Dimensional Ultrasound Assists in Accurately Characterizing Cervical Ectopic Gestation: A Case Report
}

\author{
June Ng, MD*, Shelley Dolitsky, MD, Mark V Sauer, MD and Meike Schuster, DO \\ Department of Obstetrics, Gynecology and Reproductive Sciences, Rutgers-Robert Wood Johnson Medical School, USA
}

\begin{abstract}
Cervical ectopic pregnancy (CEP) is rare, and occurs when the blastocyst implants into the endocervical canal. They are particularly dangerous because they are difficult to diagnose, and typically present with massive hemorrhage necessitating hysterectomy. Therefore, there exists a need to definitively diagnose this condition earlier.

Here, we present a case of a cervical ectopic pregnancy managed conservatively with methotrexate (MTX) once definitive diagnosis was made with three-dimensional ultrasound (3D-US). In the future, we suggest that 3D-US should be considered early on in the evaluation and imaging of pregnancies of unknown location in order to expeditiously arrive at a definitive diagnosis and allow for targeted treatment.
\end{abstract}

\section{Keywords}

Cervical ectopic pregnancy, Three-dimensional ultrasound, Methotrexate

\section{Introduction}

Cervical ectopic pregnancy (CEP) is a rare condition and occurs when the blastocyst implants into the endocervical canal. Risk factors for CEP include prior uterine surgery and assisted reproduction. Although $<1 \%$ of ectopic pregnancies implant in the cervix, they are particularly dangerous since they are difficult to diagnose and, if left untreated, typically present with massive hemorrhage [1,2]. Therefore, in order to prevent morbidity, mortality, and to preserve desired future fertility, early diagnosis of CEP is essential.

There is no consensus as to the optimal treatment for CEP, but proposed conservative medical management has included systemic methotrexate (MTX) with or without intra-amniotic instillation of MTX [3]. Rates of failure for systemic MTX are greater when beta human chorionic gonadotropin (b-hCG) levels exceed $10,000 \mathrm{mIU} / \mathrm{mL}$, gestational age is greater than 9 weeks, embryonic cardiac activity is present, or crown-rump length (CRL) is more than $10 \mathrm{~mm}$ [4]. Surgical treatment has included more conservative hysteroscopic resection, but also temporary tamponade with balloon, uterine or internal iliac artery ligation or embolization, vaginal hysterectomy, and most commonly, open hysterectomy [5-9].

Here, we present a case of a CEP that was definitively diagnosed with three-dimensional ultrasound (3D-US) and successfully managed with MTX. The superior imagery provided by 3D-US compared to conventional two-dimensional ultrasound (2D-US) should be entertained when physicians are struggling to determine whether a low-lying implantation is intrauterine or intracervical in location.

\section{Case Presentation}

A 22-year old, gravida 1, para 0 female presented at $7 \mathrm{w} 2 \mathrm{~d}$ by last menstrual period (LMP) with heavy vaginal bleeding to the emergency department (ED). Quantitative b-hCG level was noted to be $4,803 \mathrm{mlU} / \mathrm{mL}$. At the time of her initial examination, the cervix was observed to be closed with a small amount of blood oozing from the external cervical os. A 2D-US performed in the ED revealed an $8 \mathrm{~mm}$ gestational sac (GS) with a $2 \mathrm{~mm}$ yolk sac located at the junction of the upper cervix and lower uterus. Differential diagnosis included CEP or inevitable abortion and the patient was admitted for further observation.

The next morning, repeat 2D-US showed a sac with a yolk sac and crown rump length (CRL) of $2 \mathrm{~mm}$, but otherwise no changes compared to the previous day. The patient was given the first dose of a fixed multi-dose MTX protocol. This was chosen for multiple reasons. First, the patient had an initial

*Corresponding author: June Ng, MD, Department of Obstetrics, Gynecology and Reproductive Sciences, Rutgers-Robert Wood Johnson Medical School, 125 Paterson Street, New Brunswick, NJ, 08902, USA, Tel: 732-235-7633, Fax: 732-235-7035

Accepted: June 26, 2019

Published online: June 28, 2019

Citation: June NG, Dolitsky S, Sauer MV, et al. (2019) ThreeDimensional Ultrasound Assists in Accurately Characterizing Cervical Ectopic Gestation: A Case Report. Annals Gynecol Obstet 3(1):47-49 
Citation: June NG, Dolitsky S, Sauer MV, et al. (2019) Three-Dimensional Ultrasound Assists in Accurately Characterizing Cervical Ectopic Gestation: A Case Report. Annals Gynecol Obstet 3(1):47-49

high bHCG level of $4803 \mathrm{mIU} / \mathrm{mL}$ which increased to 6217 $\mathrm{mIU} / \mathrm{mL}$ on the day of first MTX administration. For ectopic pregnancies, bHCG $>5000 \mathrm{mIU} / \mathrm{mL}$ is associated with a $14.3 \%$ rate of single-dose methotrexate failure. Second, there was a strong desire from both the physician and patient to avoid surgical intervention given her nulliparous status and desire for discharge from the hospital that day. Therefore, we chose a regimen with a higher rate of efficacy; the fixed multi-dose MTX protocol is more effective than the single dose regimen (92.7\% vs. $88.1 \%$ resolution) [4]. Third, our initial investigation into the literature for MTX treatment for cervical ectopic pregnancies supported use of the fixed multi-dose regimen [10]. On that first day (Day 1), her bHCG was $6217 \mathrm{mIU} / \mathrm{mL}$. On day 3, her bHCG increased to $9381 \mathrm{mIU} / \mathrm{mL}$, and she received the second dose of intramuscular MTX.

A 2D-US was repeated on day 4 that showed a CRL of $2.6 \mathrm{~mm}$ and a fetal heart rate (FHR) of $78 \mathrm{bpm}$, which had not been seen previously. A 3D-US was then performed and confirmed the diagnosis of cervical ectopic when a ballooned cervix, closed internal os, and gestational sac within the canal, all diagnostic criteria for CEP, were clearly visualized (Figure 1). A decision was made to continue with the MTX protocol, and an additional dose of MTX was given on day 5, as prior case reports had shown a continued increase in b-HCG until after day 5 [11]. An ultrasound was repeated on day 6 and a FHR of 189 was identified. Because of the continued presence of a FHR, it was determined that the patient had failed the multi-dose MTX protocol. Therefore, a transvaginal intraamniotic sac injection of MTX was performed.

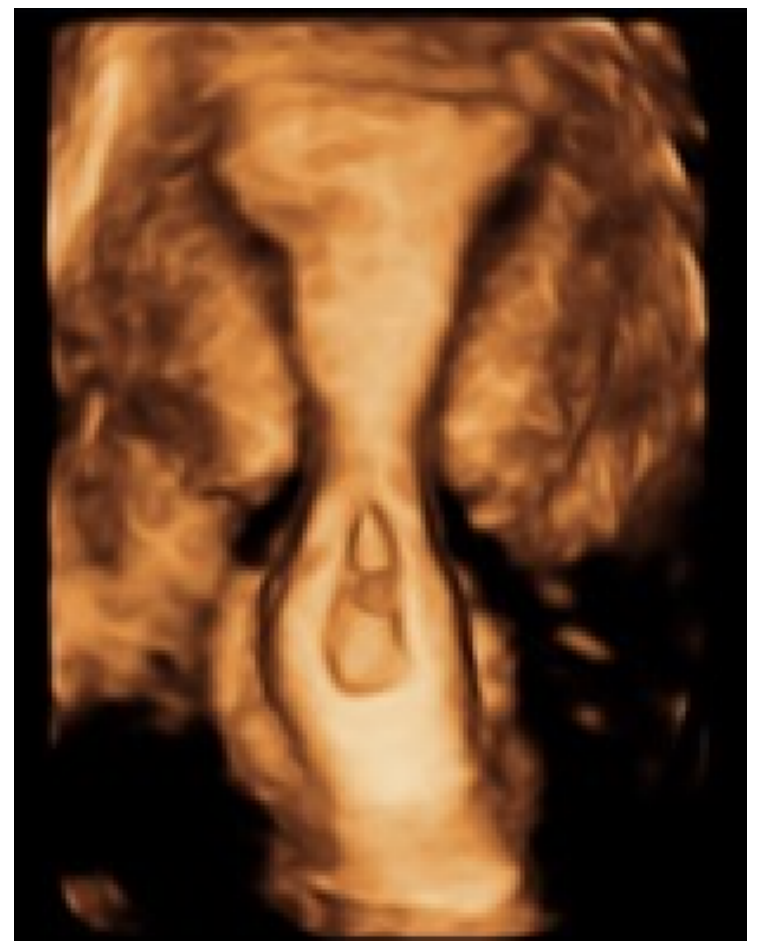

Figure 1: Three dimensional transvaginal ultrasound (3D-US) confirming diagnosis of cervical ectopic pregnancy (CEP). In this image, a ballooned cervix, closed internal os, and gestational sac within the cervix are clearly visualized.
Immediately after the procedure, FHR was $43 \mathrm{bpm}$, and prior to discharge, the FHR was $181 \mathrm{bpm}$. On day 7, her bHCG down trended to $9038 \mathrm{mIU} / \mathrm{mL}$ and by day 9 was $6929 \mathrm{mIU} /$ $\mathrm{mL}$. A repeat $2 \mathrm{D}-\mathrm{US}$ showed an irregular GS with debris and a collapsed yolk sac, no fetal pole, and no FHR. Her bHCG continued to trend down to $2320 \mathrm{mIU} / \mathrm{mL} 5$ days later and $927 \mathrm{mIU} / \mathrm{mL} 10$ days later. A scan was repeated after 4 weeks and showed absence of the yolk sac and a small collapsed gestational sac with hemorrhagic material. In summary, the patient received intramuscular MTX on days 1,3 , and 5 and intra-amniotic MTX on day 6 . She received folinic acid on days 2,4 , and 6 to minimize the adverse effects of MTX, a folic acid antagonist, per protocol by the American College of Obstetricians and Gynecologists [4]. The patient went on to resolve the pregnancy with repeat 2D-US noting no gestational sac, a normal cervix, and an endometrial stripe of $5.8 \mathrm{~mm}$.

\section{Discussion}

Differentiating between a CEP and an incomplete abortion in process can be difficult. Two-dimensional ultrasonography is traditionally used, and there are specific features that are recognized as classic for cervical ectopic gestation. To meet the criteria for cervical ectopic, there must be cervical glands opposite the placental attachment site, attachment must be below uterine vessel entry, and fetal elements must be absent from the body of the uterus. However, because this can only be diagnosed on final pathology, other clinical criteria include: A period of amenorrhea followed by bleeding without cramping, an "hourglass" cervix that is equal size or larger than the uterine corpus with closed internal os and partially open external os, and a negative "sliding organ" sign, where the sac is unable to be moved from its position within the cervix with gentle pressure from the transvaginal probe [10]. On 2D-US, an hourglass cervix and a closed internal os were suspected, but making a definitive diagnosis of cervical ectopic pregnancy and ruling out incomplete abortion remained difficult. It was only when the hourglass cervix and closed internal os were much more clearly visualized on 3D-US that a definitive diagnosis was made.

Some case reports have suggested ordering magnetic resonance imaging (MRI) to improve diagnostic accuracy. On MRI, cervical ectopic pregnancies show the "hourglass sign", a closed internal os, and a lobulated, heterogeneous, enhancing cervical mass with a T2-hypodense rim. This can be a helpful addition to 2D-US, however, MRI is much more expensive than 3D-US, takes longer to produce images, and is not always available [12]. Also, MRI images are affected by metal implants and movement, whereas ultrasound is not. In addition, MRI cannot be performed in real time and cannot use Doppler techniques to evaluate blood flow $[10,12]$. Doppler flow in particular can help quantify the degree of vascularity within a lesion as typical of an ectopic pregnancy, and using ultrasound as an extension of the physical exam in real time allows for identification of the "sliding organ" sign.

While 3D-US has been described as a possible diagnostic adjunct to diagnose CEP, it is not commonly used. The advantage of 3D-US is the ability to take coronal sections that 
Citation: June NG, Dolitsky S, Sauer MV, et al. (2019) Three-Dimensional Ultrasound Assists in Accurately Characterizing Cervical Ectopic Gestation: A Case Report. Annals Gynecol Obstet 3(1):47-49

can be generated to reconstruct views of an entire organ in any orientation and calculate volume $[13,14]$. Using 3D-US, hundreds of images can be generated to reconstruct a view of the entire organ in any orientation [14]. Without the limitations of 2D-US like orientation or rotation of the uterus, 3DUS can generate a complete evaluation of the uterus. Indeed, 3D-US has also been found in multiple studies to be similar to $M R I$ in the diagnosis of Mullerian anomalies, including cervical duplication [15]. In this patient, we were able to quickly and definitively differentiate the edges of the gestational sac, visualize the lower limit of the endometrial cavity, and make an accurate diagnosis of CEP in a timely fashion using 3D-US. Earlier implementation of 3D-US may lessen healthcare costs of by obviating the need for additional surveillance visits, employing less definitive and serially applied 2D-US, or needing an MRI to image the gestation.

In summary, we present a case of CEP that was suspected on 2D-US but definitively diagnosed with 3D-US. We suggest that 3D-US should be considered early on in the evaluation and imaging of pregnancies of unknown location in order to expeditiously arrive at a definitive diagnosis and allow for targeted treatment of abnormal pregnancies.

\section{Acknowledgements}

The authors would like to thank the medical students, residents, and faculty of Rutgers - Robert Wood Johnson Medical School, who provided support and guidance in the literature review for this case report.

\section{Declarations of Interest}

None.

\section{References}

1. Murji A, Garbedian K, Thomas J, et al. (2015) Conservative management of cervical ectopic pregnancy. J Obstet Gynaecol Can 37: 1016-1020.

2. Samal SK, Rathod S (2015) Cervical ectopic pregnancy. J Nat Sci Biol Med 6: 257-260.
3. Ushakov FB, Elchalal U, Aceman PJ, et al. (1997) Cervical pregnancy: Past and future. Obstet Gynecol Surv 52: 45-59.

4. Hung TH, Shau WY, Hsieh TT, et al. (1998) Prognostic factors for an unsatisfactory primary methotrexate treatment of cervical pregnancy: A quantitative review. Hum Reprod 13: 2636-2642.

5. Kofinas JD, Purisch SE, Brandt JS, et al. (2012) Hysteroscopic removal of cervical ectopic pregnancy following failed intramuscular/intra-sac methotrexate: A case report. J Gynecol Surg 28: 369-371.

6. Fylstra DL (2014) Cervical pregnancy: 13 cases treated with suction curettage and balloon tamponade. Am J Obstet Gynecol 210: 581.e1-581.e5.

7. Zhou Q, Young D, Vingan $H$ (2015) Uterine artery embolization for cervical ectopic pregnancy. Radiol Case Rep 10: 72-75.

8. Singh S (2013) Diagnosis and management of cervical ectopic pregnancy. J Hum Reprod Sci 6: 273-276.

9. Alammari R, Thibodeau R, Harmanli O (2017) Vaginal hysterectomy for treatment of cervical ectopic pregnancy. Obstet Gynecol 129: 63-65.

10. Dibble EH, Lourenco AP (2016) Imaging unusual pregnancy implantations: Rare ectopic pregnancies and more. AJR Am J Roentgenol 207: 1380-1392.

11. Weibel HS, Abdulrahman A, Reinhold C, et al. (2012) Multidose methotrexate treatment of cervical pregnancy. J Obstet Gynaecol Can 34: 359-362.

12. Benacerraf BR, Abuhamad AZ, Bromley B, et al. (2015) Consider ultrasound first for imaging the female pelvis. Am J Obstet Gynecol 212: 450-405.

13. Ruano R, Reya F, Picone O, et al. (2006) Three-dimensional ultrasonographic diagnosis of a cervical pregnancy. Clinics (Sao Paulo) 61: 355-358.

14. Levine EM, Fernandez CM, Miller D, et al. (2018) Clinical value of 3-dimensional ultrasound in gynecology. J Ultrasound Med 37: 2445-2450.

15. Armstrong L, Fleischer A, Andreotti R (2013) Three-dimensional volumetric sonography in gynecology: An overview of clinical applications. Radiol Clin North Am 51: 1035-1047.

DOI: $10.36959 / 468 / 465$

Copyright: (c) 2019 June NG, et al. This is an open-access article distributed under the terms of the Creative Commons Attribution License, which permits unrestricted use, distribution, and reproduction in any medium, provided the original author and source are credited. 